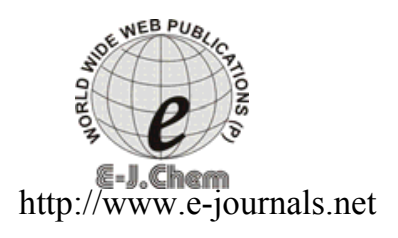

\title{
Three-Component Reaction of Triphenylphosphine, Acetylenic Esters and 4-(Arylideneamino)- 3-ethyl-1H-1,2,4-triazole-5(4H)-thiones for the Synthesis of Phosphorus Ylides
}

\author{
MOHAMMAD H. MOSSLEMIN ${ }^{\S}$, MASOUMEH TABATABAEE ${ }^{\aleph^{*}}$, \\ ALIREZA HASSANABADI and ASAL LAMEI ${ }^{\S}$ \\ ${ }^{\S}$ Department of Chemistry, Islamic Azad University \\ Yazd Branch, P.O. Box 89195-155, Yazd, Iran \\ Department of Chemistry, Islamic Azad University \\ Zahedan Branch, P.O. Box 98135-978, Zahedan, Iran \\ tabatabaee45m@yahoo.com
}

Received 20 January 2011; Accepted 1 March 2011

\begin{abstract}
Triphenylphosphine reacts with 4-(arylideneamino)-3-ethyl- $1 H$ 1,2,4-triazole-5 $(4 H)$-thiones in the presence of dialkyl acetylenedicarboxylates to produce highly functionalized, salt-free phosphorus ylides in excellent yields.
\end{abstract}

Keywords: Dialkyl acetylenedicarboxylates, Phosphorus ylides, Triphenylphosphine, NH-acid, Addition reaction

\section{Introduction}

Phosphorus ylides are reactive systems, which take part in many reactions of value in organic synthesis ${ }^{1-3}$. Several methods have been developed for the preparation of phosphorus ylides. These ylides are usually prepared by treatment of an appropriate phosphonium salt with a base; the corresponding phosphonium salts are usually obtained from the phosphine and an alkyl halide. Phosphonium salts are also prepared by Michael addition of phosphorus nucleophiles to activated olefins ${ }^{4,5}$. Reaction of acetylenic esters with triphenylphosphine in the presence of an organic compound possessing an acidic-hydrogen has been recently reported to produce phosphorus ylides ${ }^{6-16}$. In continuation of our previous works on the reaction between triphenylphosphine and acetylene esters in the presence of organic N-H, $\mathrm{O}-\mathrm{H}$ or $\mathrm{C}-\mathrm{H}$ acids ${ }^{17-23}$, we herein report an efficient synthetic route to stable phosphorus ylides using three-component condensation reaction. 


\section{Experimental}

Melting points were determined with an electrothermal 9100 apparatus. Elemental analyses were performed at Analytical Laboratory of Islamic Azad University, Yazd Branch using a Costech ECS 4010 CHNS-O analyzer. Mass spectra were recorded on a FINNIGAN-MAT 8430 mass spectrometer operating at an ionization potential of $70 \mathrm{eV}$. IR spectra were recorded on a Shimadzu IR-470 spectrometer. ${ }^{1} \mathrm{H},{ }^{13} \mathrm{C}$ and ${ }^{31} \mathrm{P}$ NMR spectra were recorded on BRUKER DRX-500 AVANCE spectrometer at 500, 128.5 and $202.5 \mathrm{MHz}$, respectively. ${ }^{1} \mathrm{H},{ }^{13} \mathrm{C}$ and ${ }^{31} \mathrm{P}$ NMR spectra were obtained on solution in $\mathrm{CDCl}_{3}$ using TMS as internal standard or $85 \% \mathrm{H}_{3} \mathrm{PO}_{4}$ as external standard. The chemicals used in this work purchased from Fluka (Buchs, Switzerland) and were used without further purification.

\section{General procedure for preparation of compounds (4a-f)}

To a magnetically stirred solution of triphenylphosphine $(2 \mathrm{mmol})$ and 4-(arylideneamino) -3-ethyl-1 $H$-1,2,4-triazole-5 $(4 H)$-thione $(2 \mathrm{mmol})$ in dichloromethane $(10 \mathrm{~mL})$ a mixture of dialkyl acetylenedicarboxylate $(2 \mathrm{mmol})$ in dichloromethane $(3 \mathrm{~mL})$ was added drop wise at room temperature over $2 \mathrm{~min}$. The reaction mixture was then stirred for $3 \mathrm{~h}$ (the progress of reaction was monitored by TLC). After completion of reaction, the solvent was evaporated at reduced pressure. The residue was precipitated in a mixture of diethyl ether-hexane. The solid was filtered and washed with diethyl ether to give the pure product.

Di-t-butyl 2-[4-(benzylidene-amino)-5-ethyl -4H -1,2,4-triazol -3 -yl sulfanyl] -3(triphenyl- $\lambda^{5}$-phosphanylidene)-succinate (4a)

Yellow powder; m.p. 129-131 ${ }^{\circ} \mathrm{C}$. IR $(\mathrm{KBr})\left(v_{\max }, \mathrm{cm}^{-1}\right): 1753(\mathrm{C}=\mathrm{O}) .{ }^{1} \mathrm{H}$ NMR $(500.1$ $\left.\mathrm{MH}_{\mathrm{Z}}, \mathrm{CDCl}_{3}\right): \delta 0.95(9 \mathrm{H}, \mathrm{s}, t-\mathrm{Bu}), 1.20\left(3 \mathrm{H}, \mathrm{t},{ }^{3} J_{\mathrm{HH}}=7 \mathrm{H}_{\mathrm{Z}}, \mathrm{CH}_{3}\right), 1.54(9 \mathrm{H}, \mathrm{s}, t-\mathrm{Bu})$, $3.47\left(2 \mathrm{H}, \mathrm{q},{ }^{3} J_{\mathrm{HH}}=7 \mathrm{H}_{\mathrm{Z}}, \mathrm{CH}_{2}\right), 5.24\left(1 \mathrm{H}, \mathrm{d},{ }^{3} J_{\mathrm{PH}}=16 \mathrm{H}_{\mathrm{Z}}\right), 7.39-7.79(20 \mathrm{H}, \mathrm{m}$, aromatic). $10.52(1 \mathrm{H}, \mathrm{s}, \mathrm{CH}=\mathrm{N}) .{ }^{13} \mathrm{C} \mathrm{NMR}\left(125.8 \mathrm{MH}_{\mathrm{Z}}, \mathrm{CDCl}_{3}\right): \delta 10.5\left(\mathrm{CH}_{3}\right), 19.4$ $\left(\mathrm{CH}_{2}\right), 28.6$ and $28.7\left(6 \mathrm{CH}_{3}\right.$ of $\left.2 t-\mathrm{Bu}\right), 38.8\left(\mathrm{~d},{ }^{1} J_{\mathrm{PC}}=130 \mathrm{H}_{\mathrm{Z}}, \mathrm{C}=\mathrm{P}\right), 63.0\left(\mathrm{~d},{ }^{2} J_{\mathrm{PC}}=16\right.$ $\left.\mathrm{H}_{\mathrm{Z}}, \mathrm{CH}\right), 81.2$ and $81.3\left(2 \mathrm{O}-\mathrm{C}\left(\mathrm{CH}_{3}\right)_{3}\right), 128.0\left(\mathrm{~d},{ }^{1} J_{\mathrm{PC}}=92 \mathrm{H}_{\mathrm{Z}}\right), 129.0\left(\mathrm{~d},{ }^{2} J_{\mathrm{PC}}=12 \mathrm{H}_{\mathrm{Z}}\right)$, $132.3\left(\mathrm{~d},{ }^{4} J_{\mathrm{PC}}=2 \mathrm{H}_{\mathrm{Z}}\right), 134.2\left(\mathrm{~d},{ }^{3} J_{\mathrm{PC}}=10 \mathrm{H}_{\mathrm{Z}}\right), 126.9,128.8,129.1,132.0(\mathrm{ph}), 151.1$ $(\mathrm{CH}=\mathrm{N}), 159.5(\mathrm{SC}=\mathrm{N}), 160.8(\mathrm{NC}=\mathrm{N}), 168.6\left(\mathrm{~d},{ }^{2} J_{\mathrm{PC}}=12 \mathrm{H}_{\mathrm{Z}}, \mathrm{C}=\mathrm{O}\right), 168.7\left(\mathrm{~d},{ }^{3} J_{\mathrm{PC}}=17\right.$ $\left.\mathrm{H}_{\mathrm{Z}}, \mathrm{C}=\mathrm{O}\right) .{ }^{31} \mathrm{P}$ NMR $\left(202.5 \mathrm{MH}_{\mathrm{Z}}, \mathrm{CDCl}_{3}\right): \delta 30.04$. Analyses: Calcd. for $\mathrm{C}_{41} \mathrm{H}_{45} \mathrm{~N}_{4} \mathrm{O}_{4} \mathrm{PS}$ : C, 68.31; H, 6.29; N,7.77. Found: C, 68.5; H, 6.1; N,7.9. MS (m/z, \%): $720\left(\mathrm{M}^{+}, 7\right) ; 262$ $\left(\mathrm{PPh}_{3}, 85\right), 77$ (Ph, 40), Yield: 93\%.

Dimethyl 2-[4-(benzylidene-amino)-5-ethyl -4H -1,2,4 -triazol-3-yl sulfanyl]3-(triphenyl- $\lambda^{5}$-phosphanylidene)-succinate (4b)

Yellow powder; m.p. 102-104 ${ }^{\circ} \mathrm{C}$. IR $(\mathrm{KBr})\left(v_{\max }, \mathrm{cm}^{-1}\right)$ : $1751(\mathrm{C}=\mathrm{O}) .{ }^{1} \mathrm{H}$ NMR $(500.1$ $\left.\mathrm{MHz}, \mathrm{CDCl}_{3}\right): \delta 1.20\left(3 \mathrm{H}, \mathrm{t},{ }^{3} \mathrm{~J}_{\mathrm{HH}}=7 \mathrm{H}_{\mathrm{Z}}, \mathrm{CH}_{3}\right), 3.14\left(3 \mathrm{H}, \mathrm{s}, \mathrm{OCH}_{3}\right), 3.52(2 \mathrm{H}, \mathrm{q}$, $\left.{ }^{3} J_{\mathrm{HH}}=7 \mathrm{H}_{\mathrm{Z}}, \mathrm{CH}_{2}\right), 3.79\left(3 \mathrm{H}, \mathrm{s}, \mathrm{OCH}_{3}\right), 5.58\left(1 \mathrm{H}, \mathrm{d},{ }^{3} J_{\mathrm{PH}}=16 \mathrm{H}_{\mathrm{Z}}\right), 7.43-7.85(20 \mathrm{H}$, $\mathrm{m}$, aromatic), $10.49(1 \mathrm{H}, \mathrm{s}, \mathrm{CH}=\mathrm{N}) .{ }^{13} \mathrm{C} \mathrm{NMR}\left(125.8 \mathrm{MHz}, \mathrm{CDCl}_{3}\right): \delta 11.0\left(\mathrm{CH}_{3}\right), 19.4$ $\left(\mathrm{CH}_{2}\right), 38.9\left(\mathrm{~d},{ }^{1} J_{\mathrm{PC}}=130 \mathrm{H}_{\mathrm{Z}}, \mathrm{C}=\mathrm{P}\right), 49.7\left(\mathrm{OCH}_{3}\right), 53.1\left(\mathrm{OCH}_{3}\right), 62.4\left(\mathrm{~d},{ }^{2} J_{\mathrm{PC}}=16 \mathrm{H}_{\mathrm{Z}}, \mathrm{CH}\right)$, $127.1\left(\mathrm{~d},{ }^{1} J_{\mathrm{PC}}=92 \mathrm{H}_{\mathrm{Z}}\right), 129.2\left(\mathrm{~d},{ }^{2} J_{\mathrm{PC}}=12 \mathrm{H}_{\mathrm{Z}}\right), 132.4\left(\mathrm{~d},{ }^{4} J_{\mathrm{PC}}=2 \mathrm{H}_{\mathrm{Z}}\right), 134.2\left(\mathrm{~d},{ }^{3} J_{\mathrm{PC}}=10\right.$ $\left.\mathrm{H}_{\mathrm{Z}}\right), 126.1,128.9,129.3,132.3(\mathrm{ph}), 151.2(\mathrm{CH}=\mathrm{N}), 159.6(\mathrm{SC}=\mathrm{N}), 161.1(\mathrm{NC}=\mathrm{N}), 168.5$ $\left(\mathrm{d},{ }^{2} J_{\mathrm{PC}}=12 \mathrm{H}_{\mathrm{Z}} \mathrm{C}=\mathrm{O}\right), 170.8\left(\mathrm{~d},{ }^{3} J_{\mathrm{PC}}=17 \mathrm{H}_{\mathrm{Z}} \mathrm{C}=\mathrm{O}\right) .{ }^{31} \mathrm{P}$ NMR $\left(202.5 \mathrm{MHz}, \mathrm{CDCl}_{3}\right): \delta$ 30.07. Analyses: Calcd. for $\mathrm{C}_{35} \mathrm{H}_{33} \mathrm{~N}_{4} \mathrm{O}_{4} \mathrm{PS}: \mathrm{C}, 66.02 ; \mathrm{H}, 5.22 ; \mathrm{N}, 8.80$. Found: $\mathrm{C}, 66.1 ; \mathrm{H}$, 5.3; N, 8.9. MS (m/z, \%): $636\left(\mathrm{M}^{+}, 5\right) ; 262\left(\mathrm{PPh}_{3}, 80\right), 77$ (Ph, 55), Yield: 91\%. 
Diethyl 2-[4-(benzylidene - amino) - 5- ethyl-4H-1,2,4 -triazol -3-yl sulfanyl] 3-(triphenyl- $\lambda^{5}$-phosphanylidene)-succinate (4c)

Yellow powder; m.p. $116-118{ }^{\circ} \mathrm{C}$. IR $(\mathrm{KBr})\left(v_{\max }, \mathrm{cm}^{-1}\right): 1748(\mathrm{C}=\mathrm{O}) .{ }^{1} \mathrm{H}$ NMR $(500.1 \mathrm{MHz}$, $\left.\mathrm{CDCl}_{3}\right): \delta 1.17\left(3 \mathrm{H}, \mathrm{t},{ }^{3} J_{\mathrm{HH}}=7 \mathrm{H}_{\mathrm{Z}}, \mathrm{CH}_{3}\right), 1.24\left(3 \mathrm{H}, \mathrm{t},{ }^{3} J_{\mathrm{HH}}=7 \mathrm{H}_{\mathrm{Z}}, \mathrm{CH}_{3}\right), 1.36(3 \mathrm{H}, \mathrm{t}$, $\left.{ }^{3} J_{\mathrm{HH}}=7 \mathrm{H}_{\mathrm{Z}}, \mathrm{CH}_{3}\right), 3.47\left(2 \mathrm{H}, \mathrm{q},{ }^{3} J_{\mathrm{HH}}=7 \mathrm{H}_{\mathrm{Z}}, \mathrm{CH}_{2}\right), 4.07\left(2 \mathrm{H}, \mathrm{q},{ }^{3} J_{\mathrm{HH}}=7 \mathrm{H}_{\mathrm{Z}}, \mathrm{OCH}_{2}\right), 4.25$ $\left(2 \mathrm{H}, \mathrm{q},{ }^{3} \mathrm{~J}_{\mathrm{HH}}=7 \mathrm{H}_{\mathrm{Z}}, \mathrm{OCH}_{2}\right), 5.75\left(1 \mathrm{H}, \mathrm{d},{ }^{3} \mathrm{~J}_{\mathrm{PH}}=16 \mathrm{H}_{\mathrm{Z}}, \mathrm{CH}\right), 7.45-7.87$ (20H, m, aromatic), $10.50(1 \mathrm{H}, \mathrm{s}, \mathrm{CH}=\mathrm{N}) \cdot{ }^{13} \mathrm{C} \mathrm{NMR}\left(125.8 \mathrm{MHz}, \mathrm{CDCl}_{3}\right): \delta 10.9\left(\mathrm{CH}_{3}\right), 14.6$ and $14.7\left(2 \mathrm{CH}_{3}\right)$, $19.4\left(\mathrm{CH}_{2}\right), 40.4\left(\mathrm{~d},{ }^{1} J_{\mathrm{PC}}=130 \mathrm{H}_{\mathrm{Z}}, \mathrm{C}=\mathrm{P}\right), 61.7\left(\mathrm{~d},{ }^{2} J_{\mathrm{PC}}=16 \mathrm{H}_{\mathrm{Z}}, \mathrm{CH}\right), 58.2$ and 58.9 $\left(2 \mathrm{OCH}_{2}\right), 126.9\left(\mathrm{~d},{ }^{1} J_{\mathrm{PC}}=92 \mathrm{H}_{\mathrm{Z}}\right), 128.9\left(\mathrm{~d},{ }^{2} J_{\mathrm{PC}}=12 \mathrm{H}_{\mathrm{Z}}\right), 132.4\left(\mathrm{~d},{ }^{4} J_{\mathrm{PC}}=2 \mathrm{H}_{\mathrm{Z}}\right), 134.2$ $\left(\mathrm{d},{ }^{3} J_{\mathrm{PC}}=10 \mathrm{H}_{\mathrm{Z}}\right), 126.1,128.8,129.2,132.2(\mathrm{ph}), 151.2(\mathrm{CH}=\mathrm{N}), 159.6(\mathrm{SC}=\mathrm{N}), 161.0$ $(\mathrm{NC}=\mathrm{N}), 169.7\left(\mathrm{~d},{ }^{2} J_{\mathrm{PC}}=12 \mathrm{H}_{\mathrm{Z}}, \mathrm{C}=\mathrm{O}\right), 170.0\left(\mathrm{~d},{ }^{3} J_{\mathrm{PC}}=17 \mathrm{H}_{\mathrm{Z}}, \mathrm{C}=\mathrm{O}\right) .{ }^{31} \mathrm{P}$ NMR $(202.5 \mathrm{MHz}$, $\mathrm{CDCl}_{3}$ ): $\delta$ 29.98. Analyses: Calcd. for $\mathrm{C}_{37} \mathrm{H}_{37} \mathrm{~N}_{4} \mathrm{O}_{4} \mathrm{PS}: \mathrm{C}, 66.85 ; \mathrm{H}, 5.61 ; \mathrm{N}, 8.43$. Found: $\mathrm{C}$, 66.9; H, 5.5; N,8.5. MS (m/z, \%): $664\left(\mathrm{M}^{+}, 8\right) ; 262\left(\mathrm{PPh}_{3}, 87\right), 77$ (Ph, 55), Yield: 89\%.

Dimethyl 2-\{4 - [ (4- chloro-benzylidene) -amino] -5- ethyl-4H- 1,2,4 -triazol -3yl sulfanyl\}-3-(triphenyl- $\lambda^{5}$-phosphanylidene)-succinate (4d)

Yellow powder; m.p. $195-197{ }^{\circ} \mathrm{C}$. IR $(\mathrm{KBr})\left(v_{\max }, \mathrm{cm}^{-1}\right)$ : $1745(\mathrm{C}=\mathrm{O}) .{ }^{1} \mathrm{H}$ NMR $(500.1 \mathrm{MHz}$, $\left.\mathrm{CDCl}_{3}\right): \delta 1.19\left(3 \mathrm{H}, \mathrm{t},{ }^{3} J_{\mathrm{HH}}=7 \mathrm{H}_{\mathrm{Z}}, \mathrm{CH}_{3}\right), 3.24\left(3 \mathrm{H}, \mathrm{s}, \mathrm{OCH}_{3}\right), 3.50\left(2 \mathrm{H}, \mathrm{q},{ }^{3} J_{\mathrm{HH}}=7 \mathrm{H}_{\mathrm{Z}}\right.$, $\left.\mathrm{CH}_{2}\right), 3.75\left(3 \mathrm{H}, \mathrm{s}, \mathrm{OCH}_{3}\right), 5.52\left(1 \mathrm{H}, \mathrm{d},{ }^{3} \mathrm{~J}_{\mathrm{PH}}=16 \mathrm{H}_{\mathrm{Z}}\right), 7.59-7.95(19 \mathrm{H}, \mathrm{m}$, aromatic), $10.05(1 \mathrm{H}, \mathrm{s}, \mathrm{CH}=\mathrm{N}) .{ }^{13} \mathrm{C}$ NMR $\left(125.8 \mathrm{MHz}, \mathrm{CDCl}_{3}\right): \delta 9.5\left(\mathrm{CH}_{3}\right), 17.8\left(\mathrm{CH}_{2}\right), 38.8\left(\mathrm{~d},{ }^{1} J_{\mathrm{PC}}\right.$ $\left.=130 \mathrm{H}_{\mathrm{Z}}, \mathrm{C}=\mathrm{P}\right), 51.3\left(\mathrm{OCH}_{3}\right), 51.7\left(\mathrm{OCH}_{3}\right), 61.9\left(\mathrm{~d},{ }^{2} J_{\mathrm{PC}}=16 \mathrm{H}_{\mathrm{Z}} \mathrm{CH}\right), 127.9\left(\mathrm{~d},{ }^{1} J_{\mathrm{PC}}=92\right.$ $\left.\mathrm{H}_{\mathrm{Z}}\right), 129.2\left(\mathrm{~d},{ }^{2} J_{\mathrm{PC}}=12 \mathrm{H}_{\mathrm{Z}}\right), 132.5\left(\mathrm{~d},{ }^{4} J_{\mathrm{PC}}=2 \mathrm{H}_{\mathrm{Z}}\right), 133.9\left(\mathrm{~d},{ }^{3} J_{\mathrm{PC}}=10 \mathrm{H}_{\mathrm{Z}}\right), 128.8,129.6$, $132.2,136.7(\mathrm{ph}), 151.8(\mathrm{CH}=\mathrm{N}), 160.8(\mathrm{SC}=\mathrm{N}), 161.1(\mathrm{NC}=\mathrm{N}), 168.9\left(\mathrm{~d},{ }^{2} J_{\mathrm{PC}}=12 \mathrm{H}_{\mathrm{Z}}\right.$ $\mathrm{C}=\mathrm{O}), 170.2\left(\mathrm{~d},{ }^{3} J_{\mathrm{PC}}=17 \mathrm{H}_{\mathrm{Z}} \mathrm{C}=\mathrm{O}\right) .{ }^{31} \mathrm{P}$ NMR $\left(202.5 \mathrm{MHz}, \mathrm{CDCl}_{3}\right): \delta 29.92$. Analyses: Calcd. for $\mathrm{C}_{35} \mathrm{H}_{32} \mathrm{ClN}_{4} \mathrm{O}_{4} \mathrm{PS}$ : C, 62.64; H, 4.81; N, 8.35. Found: C, 62.7; H, 4.9; N, 8.4. MS (m/z, \%): $670\left(\mathrm{M}^{+}, 7\right) ; 262\left(\mathrm{PPh}_{3}, 88\right), 77$ (Ph, 48), Yield: 90\%.

Diethyl 2-\{4-[ (4-chloro-benzylidene) - amino] -5 -ethyl-4H- 1,2,4-triazol -3-yl sulfanyl\}-3-(triphenyl- $\lambda^{5}-$ phosphanylidene)-succinate (4e)

Yellow powder; m.p. $103-105{ }^{\circ} \mathrm{C}$. IR $(\mathrm{KBr})\left(v_{\max }, \mathrm{cm}^{-1}\right)$ : $1741(\mathrm{C}=\mathrm{O}) .{ }^{1} \mathrm{H}$ NMR $(500.1 \mathrm{MHz}$, $\left.\mathrm{CDCl}_{3}\right): \delta 1.05\left(3 \mathrm{H}, \mathrm{t},{ }^{3} J_{\mathrm{HH}}=7 \mathrm{H}_{\mathrm{Z}}, \mathrm{CH}_{3}\right), 1.21\left(3 \mathrm{H}, \mathrm{t},{ }^{3} J_{\mathrm{HH}}=7 \mathrm{H}_{\mathrm{Z}}, \mathrm{CH}_{3}\right), 1.39(3 \mathrm{H}, \mathrm{t}$, $\left.{ }^{3} J_{\mathrm{HH}}=7 \mathrm{H}_{\mathrm{Z}}, \mathrm{CH}_{3}\right), 3.49\left(2 \mathrm{H}, \mathrm{q},{ }^{3} J_{\mathrm{HH}}=7 \mathrm{H}_{\mathrm{Z}}, \mathrm{CH}_{2}\right), 3.94\left(2 \mathrm{H}, \mathrm{q},{ }^{3} J_{\mathrm{HH}}=7 \mathrm{H}_{\mathrm{Z}}, \mathrm{OCH}_{2}\right), 4.18$ $\left(2 \mathrm{H}, \mathrm{q},{ }^{3} J_{\mathrm{HH}}=7 \mathrm{H}_{\mathrm{Z}}, \mathrm{OCH}_{2}\right), 5.71\left(1 \mathrm{H}, \mathrm{d},{ }^{3} \mathrm{~J}_{\mathrm{PH}}=16 \mathrm{H}_{\mathrm{Z}}, \mathrm{CH}\right), 7.57-7.95(19 \mathrm{H}, \mathrm{m}$, aromatic), $9.96(1 \mathrm{H}, \mathrm{s}, \mathrm{CH}=\mathrm{N}) \cdot{ }^{13} \mathrm{C} \mathrm{NMR}\left(125.8 \mathrm{MHz}, \mathrm{CDCl}_{3}\right): \delta 9.5\left(\mathrm{CH}_{3}\right), 13.3$ and $13.4\left(2 \mathrm{CH}_{3}\right)$, $17.7\left(\mathrm{CH}_{2}\right), 40.2\left(\mathrm{~d},{ }^{1} J_{\mathrm{PC}}=130 \mathrm{H}_{\mathrm{Z}}, \mathrm{C}=\mathrm{P}\right), 61.6\left(\mathrm{~d},{ }^{2} J_{\mathrm{PC}}=16 \mathrm{H}_{\mathrm{Z}}, \mathrm{CH}\right), 58.1$ and 58.9 $\left(2 \mathrm{OCH}_{2}\right), 127.9\left(\mathrm{~d},{ }^{1} J_{\mathrm{PC}}=92 \mathrm{H}_{\mathrm{Z}}\right), 128.9\left(\mathrm{~d},{ }^{2} J_{\mathrm{PC}}=12 \mathrm{H}_{\mathrm{Z}}\right), 132.3\left(\mathrm{~d},{ }^{4} J_{\mathrm{PC}}=2 \mathrm{H}_{\mathrm{Z}}\right), 134.2$ $\left(\mathrm{d},{ }^{3} J_{\mathrm{PC}}=10 \mathrm{H}_{\mathrm{Z}}\right), 128.8,129.7,132.1,136.8(\mathrm{ph}), 151.8(\mathrm{CH}=\mathrm{N}) 161.0(\mathrm{SC}=\mathrm{N}), 161.3$ $(\mathrm{NC}=\mathrm{N}), 169.6\left(\mathrm{~d},{ }^{2} J_{\mathrm{PC}}=12 \mathrm{~Hz}, \mathrm{C}=\mathrm{O}\right), 169.9\left(\mathrm{~d},{ }^{3} J_{\mathrm{PC}}=17 \mathrm{H}, \mathrm{C}=\mathrm{O}\right) .{ }^{31} \mathrm{P}$ NMR $(202.5 \mathrm{MHz}$, $\mathrm{CDCl}_{3}$ ): $\delta$ 29.95. Analyses: Calcd. for $\mathrm{C}_{37} \mathrm{H}_{36} \mathrm{ClN}_{4} \mathrm{O}_{4} \mathrm{PS}$ : C, 63.56; H, 5.19; N, 8.01. Found: C, 63.6; H, 5.1; N, 8.1. MS (m/z, \%): $698\left(\mathrm{M}^{+}, 6\right) ; 262\left(\mathrm{PPh}_{3}, 80\right), 77$ (Ph, 50), Yield: 87\%.

Di-t-butyl 2-\{4 -[ ( 4 -chloro - benzylidene ) -amino] -5-ethyl -4H- 1,2,4 - triazol3-yl sulfanyl\} -3-(triphenyl $-\lambda^{5}-$ phosphanylidene) -succinate (4f)

Yellow powder; m.p. $151-153{ }^{\circ} \mathrm{C}$. IR $(\mathrm{KBr})\left(v_{\max }, \mathrm{cm}^{-1}\right)$ : $1743(\mathrm{C}=\mathrm{O}) .{ }^{1} \mathrm{H}$ NMR $\left(500.1 \mathrm{MH}_{\mathrm{Z}}\right.$, $\left.\mathrm{CDCl}_{3}\right): \delta \quad 0.94(9 \mathrm{H}, \mathrm{s}, t-\mathrm{Bu}), 1.20\left(3 \mathrm{H}, \mathrm{t},{ }^{3} J_{\mathrm{HH}}=7 \mathrm{H}_{\mathrm{Z}}, \mathrm{CH}_{3}\right), 1.54(9 \mathrm{H}, \mathrm{s}, t-\mathrm{Bu}), 3.48$ $\left(2 \mathrm{H}, \mathrm{q},{ }^{3} J_{\mathrm{HH}}=7 \mathrm{H}_{\mathrm{Z}}, \mathrm{CH}_{2}\right), 5.22\left(1 \mathrm{H}, \mathrm{d},{ }^{3} J_{\mathrm{PH}}=16 \mathrm{H}_{\mathrm{Z}}\right), 7.38-7.80(19 \mathrm{H}, \mathrm{m}$, aromatic $) .10 .62$ $(1 \mathrm{H}, \mathrm{s}, \mathrm{CH}=\mathrm{N}) .{ }^{13} \mathrm{C} \mathrm{NMR}\left(125.8 \mathrm{MH}_{\mathrm{Z}}, \mathrm{CDCl}_{3}\right): \delta 10.5\left(\mathrm{CH}_{3}\right), 19.4\left(\mathrm{CH}_{2}\right), 28.6$ and 28.7 $\left(6 \mathrm{CH}_{3}\right.$ of $\left.2 t-\mathrm{Bu}\right), 38.7\left(\mathrm{~d},{ }^{1} J_{\mathrm{PC}}=130 \mathrm{H}_{\mathrm{Z}} \mathrm{C}=\mathrm{P}\right), 63.0\left(\mathrm{~d},{ }^{2} J_{\mathrm{PC}}=16 \mathrm{H}_{\mathrm{Z}}, \mathrm{CH}\right), 81.2$ and $81.3(2 \mathrm{O}-\mathrm{C}$ 
$\left.\left(\mathrm{CH}_{3}\right)_{3}\right), 127.9\left(\mathrm{~d},{ }^{1} J_{\mathrm{PC}}=92 \mathrm{H}_{\mathrm{Z}}\right), 128.9\left(\mathrm{~d},{ }^{2} J_{\mathrm{PC}}=12 \mathrm{H}_{\mathrm{Z}}\right), 132.3\left(\mathrm{~d},{ }^{4} J_{\mathrm{PC}}=2 \mathrm{H}_{\mathrm{Z}}\right), 134.2$ $\left(\mathrm{d},{ }^{3} J_{\mathrm{PC}}=10 \mathrm{H}_{\mathrm{Z}}\right), 129.5,129.9,132.2,138.0(\mathrm{ph}), 151.0(\mathrm{CH}=\mathrm{N}), 159.5(\mathrm{SC}=\mathrm{N}), 160.8$ $(\mathrm{NC}=\mathrm{N}), 168.5\left(\mathrm{~d},{ }^{2} J_{\mathrm{PC}}=12 \mathrm{H}_{\mathrm{Z}}, \mathrm{C}=\mathrm{O}\right), 168.7\left(\mathrm{~d},{ }^{3} J_{\mathrm{PC}}=17 \mathrm{H}_{\mathrm{Z}}, \mathrm{C}=\mathrm{O}\right) .{ }^{31} \mathrm{P}$ NMR $\left(202.5 \mathrm{MH}_{\mathrm{Z}}\right.$, $\mathrm{CDCl}_{3}$ ): $\delta$ 29.88. Analyses: Calcd. for $\mathrm{C}_{41} \mathrm{H}_{44} \mathrm{ClN}_{4} \mathrm{O}_{4} \mathrm{PS}: \mathrm{C}, 65.20 ; \mathrm{H}, 5.87 ; \mathrm{N}, 7.42$. Found: C, 65.3; H, 5.9; N, 7.5. MS (m/z, \%): $754\left(\mathrm{M}^{+}, 8\right) ; 262\left(\mathrm{PPh}_{3}, 90\right), 77$ (Ph, 42), Yield: 90\%.

\section{Results and Discussion}

After some preliminary experiments, it was found that a mixture of triphenylphosphine (1), 4-(arylideneamino)-3-ethyl-1 $H$-1,2,4-triazole-5(4H)-thiones (2a,b) and dialkyl acetylenedicarboxylates (DAAD) (3a-c) afforded corresponding products (4a-f) in good yields (Figure 1).<smiles>[R]OC(=O)C#CC([R])([R])[R]</smiles>

3

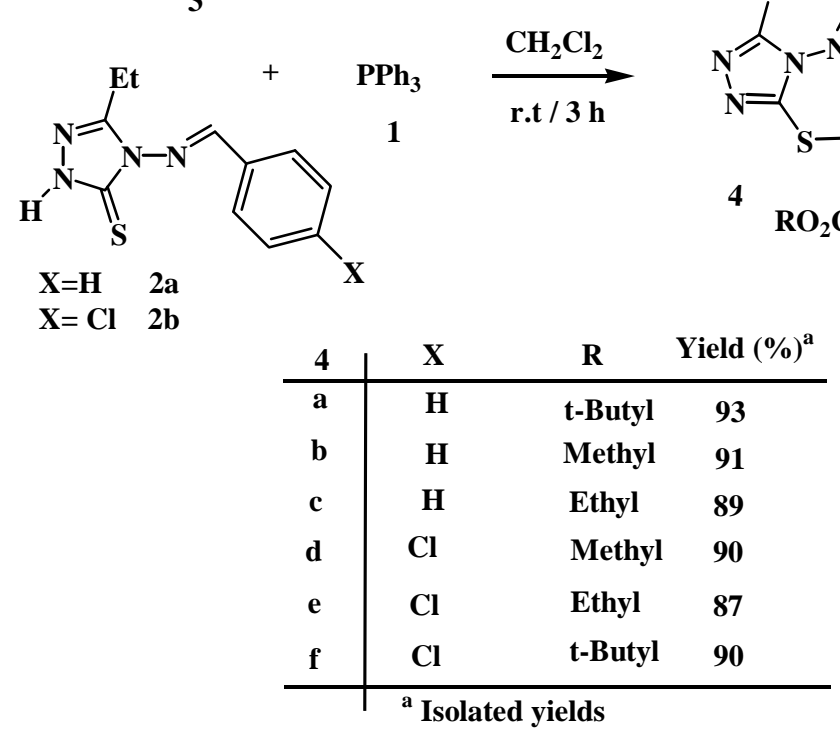

Figure 1. Three-component reaction between dialkyl acetylenedicarboxylate, triphenylphosphine and 4-(arylideneamino)-3-ethyl-1 $H$ - 1,2,4-triazole-5(4H)-thiones

The nature of these compounds as 1:1:1 adducts was apparent from their mass spectra, which displayed, in each case, the molecular ion peak at appropriate $m / z$ values. The ${ }^{1} \mathrm{H}$ NMR spectrum of compound $4 \mathrm{a}$ displays two sharp lines $(\delta 0.95,1.54 \mathrm{ppm})$ for the protons of two t-butyl groups, a doubled signal for the methine proton at $5.24 \mathrm{ppm}\left({ }^{3} J_{\mathrm{HP}}=16 \mathrm{H}_{\mathrm{Z}}\right)$, a single signal at $10.52 \mathrm{ppm}$ for the $\mathrm{C} H=\mathrm{N}$ proton and multiplets for aromatic protons $(\delta 7.39-7.79 \mathrm{ppm}) .{ }^{13} \mathrm{C}$ NMR spectrum of compound 4a showed twenty one distinct signals, which is consistent with the proposed structure. The ${ }^{31} \mathrm{P}$ NMR spectrum of compound 4 a consists of one signal at $30.04 \mathrm{ppm}$. This shift is similar to those observed for other stable phosphorus compounds ${ }^{24}$. The structural assignments made on the basis of the NMR spectra of compounds 4a-f are supported by their IR spectra. The carbonyl region of the spectrum exhibits absorption bands at $1753 \mathrm{~cm}^{-1}$ for the ester groups.

It is reasonable to assume that formation of $\mathbf{4}$ results from the initial addition of triphenylphosphine (1) to DAAD (3) and subsequent protonation of the 1:1 adduct by the $\mathrm{NH}$-acidic triazole. The positively charged ion $\mathbf{5}$ is then attacked by the triazole anion $\mathbf{6}$ to form the phosphorane 4 (Figure 2). 


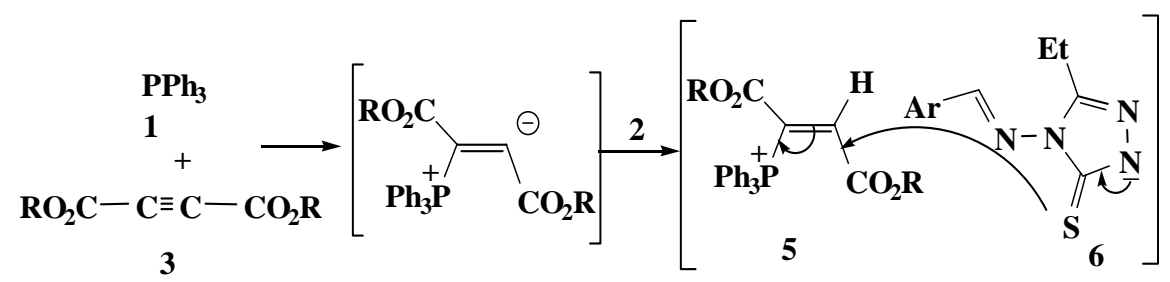

Figure 2. Suggested mechanism for formation of ylides (4)

\section{Conclusion}

We have described a simple, and one-pot, three-component reaction between dialkyl acetylenedicarboxylate, triphenylphosphine and 4-(arylideneamino)-3-ethyl-1H-1,2,4triazole-5 $(4 H)$-thiones for the preparation of functionalized phosphoranes in good yields. The advantages of the reported method are simple available starting materials, short reaction time, simple work-up, neutral reaction conditions and high yields.

\section{Acknowledgement}

We gratefully acknowledge financial support from the Research Council of Islamic Azad University, Yazd branch and the Islamic Azad University, Zahedan branch.

\section{References}

1 Kolodiazhnyi O I, Russ Chem Rev.,. 1997, 66, 225.

2 Bestmann H J and Zimmermann R, Top Curr Chem., 1983, 109, 85.

3 Maryano B E and Reits A B, Chem Rev., 1989, 89, 863.

4 Engel R, Synthesis of Carbon-Phosphorus Bonds (CRC Press, Boca Raton, FL. 1988).

5 Corbridge D E C, Phosphorus, An Outline of Chemistry, Biochemistry and Uses; $5^{\text {th }}$ Edn., Elsevier, Amsterdam, 1995.

6 Yavari I and Karimi E, Phosphorus Sulfur and Silicon Relat Elem., 2007, 182, 595.

7 Islami M R, Amrollahi M A and Iranmanesh M, Arkivoc, 2009, 10, 35-42.

8 Alizadeh A, Zohreh N and Zhu L G, Synthesis, 2009, 3, 464.

9 Maghsoodlou M T, Hazeri N, Khorasani H M, Mahmoudi Moghaddam H, Nassiri M and Salehzadeh J, Phosphorus Sulfur and Silicon Relat Elem., 2009, 184(7), 1713.

10 Maghsoodlou M T, Khorasani H M, Heydari R, Hassankhani A, Marandi G, Nassiri M and Mosaddeg E, Mol Divers., 2007, 11(2), 87-91.

11 Islami M R, Sheibani H, Hosseininasab F A and Hassanpour A, Phosphorus Sulfur and Silicon Relat Elem., 2007, 182, 1003-1010.

12 Khabazzadeh H, Saidi K, Sheibani H and Islami M R, Phosphorus Sulfur and Silicon Relat Elem., 2007, 182, 2163-2170.

13 Yavari I, Alizadeh A and Anary-Abbasinejad M, Phosphorus, Sulfur and Silicon Relat Elem., 2003, 178, 269.

14 Yavari I and Zabarjad-Shiraz N, Monatshe Chem., 2003, 134, 445-451.

15 Hassani Z, Islami M R, Moradi A and Yazdi Rouh-Alamini M, Phosphorus Sulfur and Silicon Relat Elem., 2009, 184, 568-577.

16 Esmaili A A, Ghereghloo M, Islami M R and Bijanzadeh H. R, Tetrahedron, 2003, 59, 4785-4788.

17 Hassanabadi A, Anary-Abbasinejad M and Dehghan A, Synth Commun., 2009, 39, 132.

18 Anary-Abbasinejad M, Mosslemin M H, Hassanabadi A and Tabatabaee M, Synth Commun., 2008, 38, 3700-3705. 
19 Anary-Abbasinejad M, Anaraki-Ardakani H and Hosseini-Mehdiabad H, Phosphorus Sulfur and Silicon Relat Elem., 2008, 183, 1440.

20 Anaraki-Ardakani H, Sadeghian S, Rastegari F, Hassanabadi A and AnaryAbbasinejad M, Synth Commun., 2008, 38, 1990.

21 Anary-Abbasinejad M, Anaraki-Ardakani H, Dehghan A, Hassanabadi A and Seyedmir M R, J Chem Res., 2007, 574-576.

22 Anary-Abbasinejad M, Anaraki-Ardakani H, Ezadi A and Hassanabadi A, $J$ Chem Res., 2007, 605.

23 Mosslemin M H, Anary-Abbasinejad M, Hassanabadi A and Bagheri M A, J Sulfur Chem., 2010, 31, 135-139.

24 Vedejs E and Snoble K A J, J Am Chem Soc., 1973, 95, 5778. 


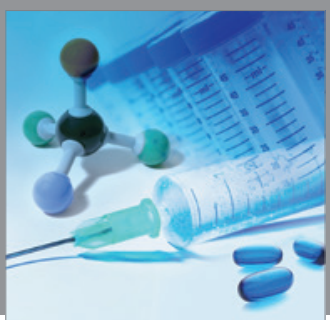

International Journal of

Medicinal Chemistry

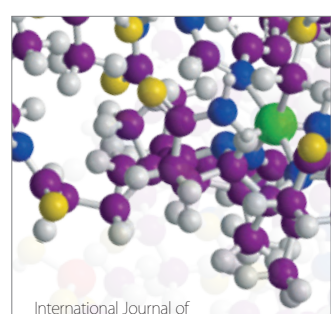

Carbohydrate Chemistry

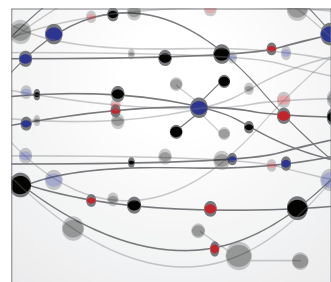

The Scientific World Journal
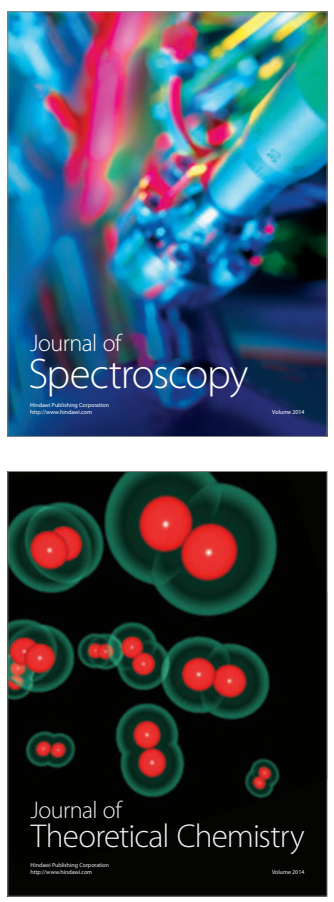
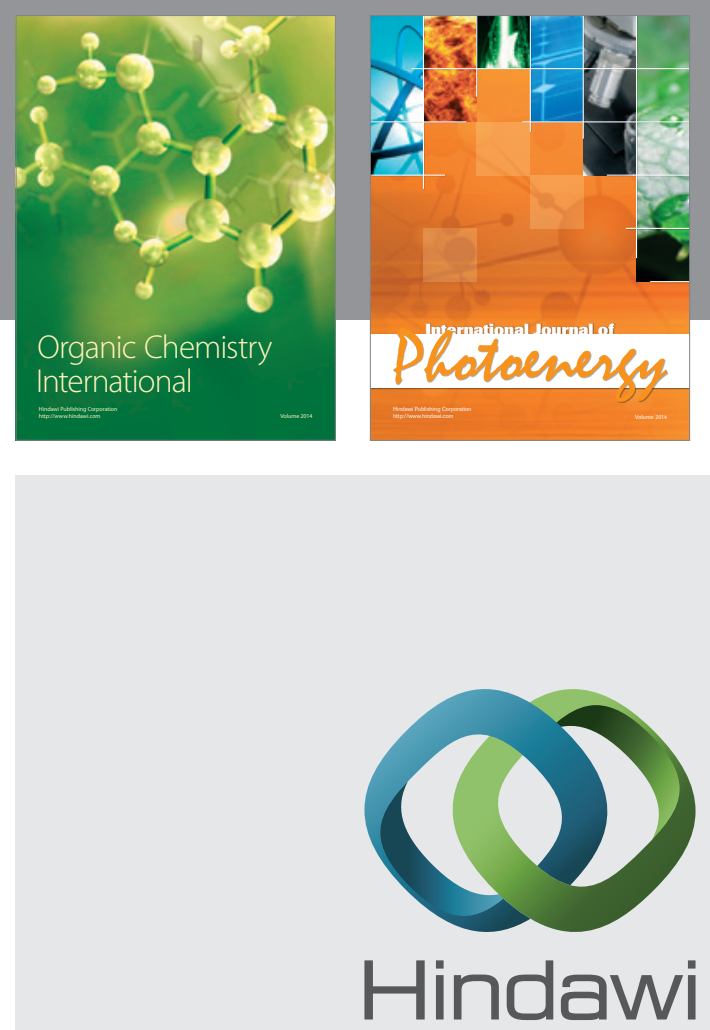

Submit your manuscripts at

http://www.hindawi.com
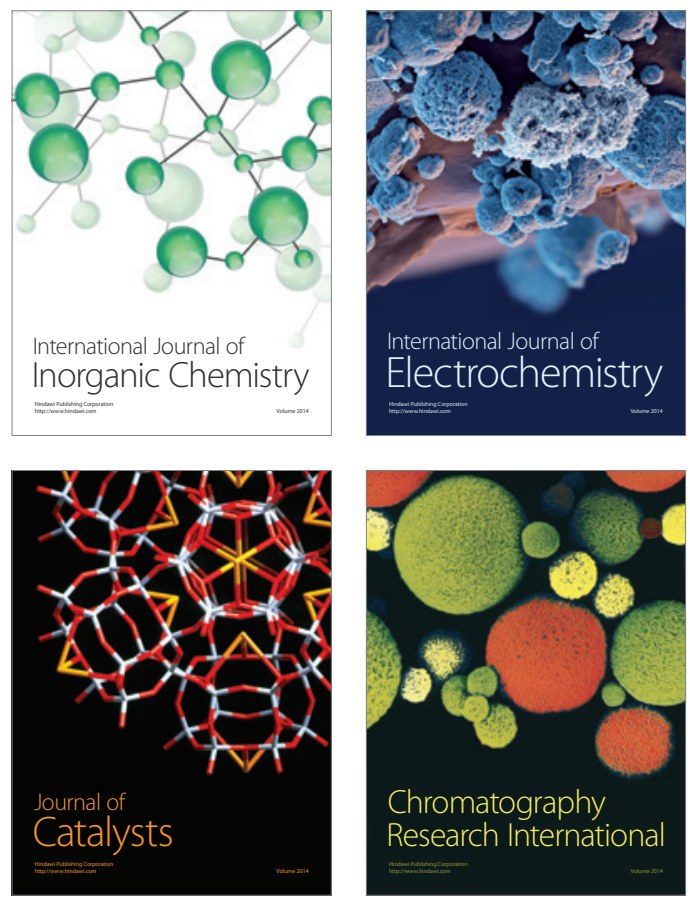
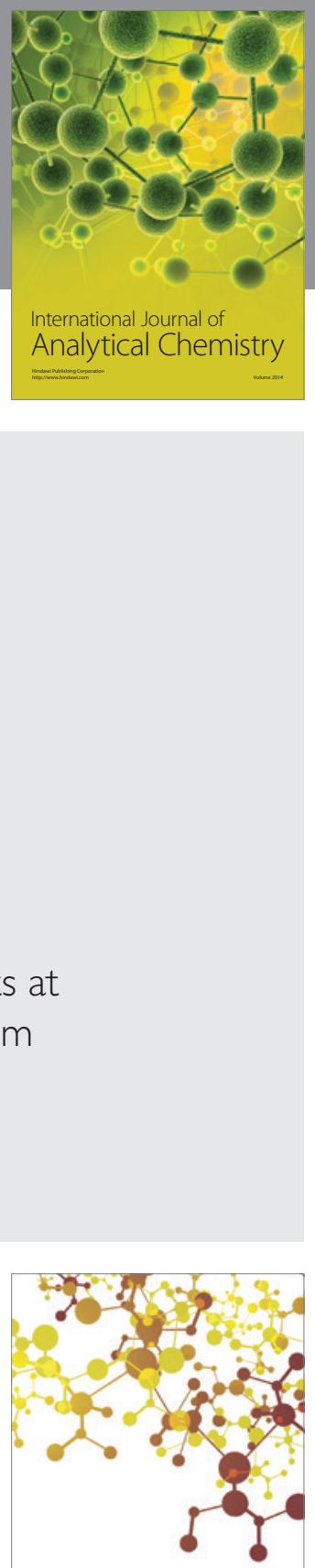

Journal of

Applied Chemistry
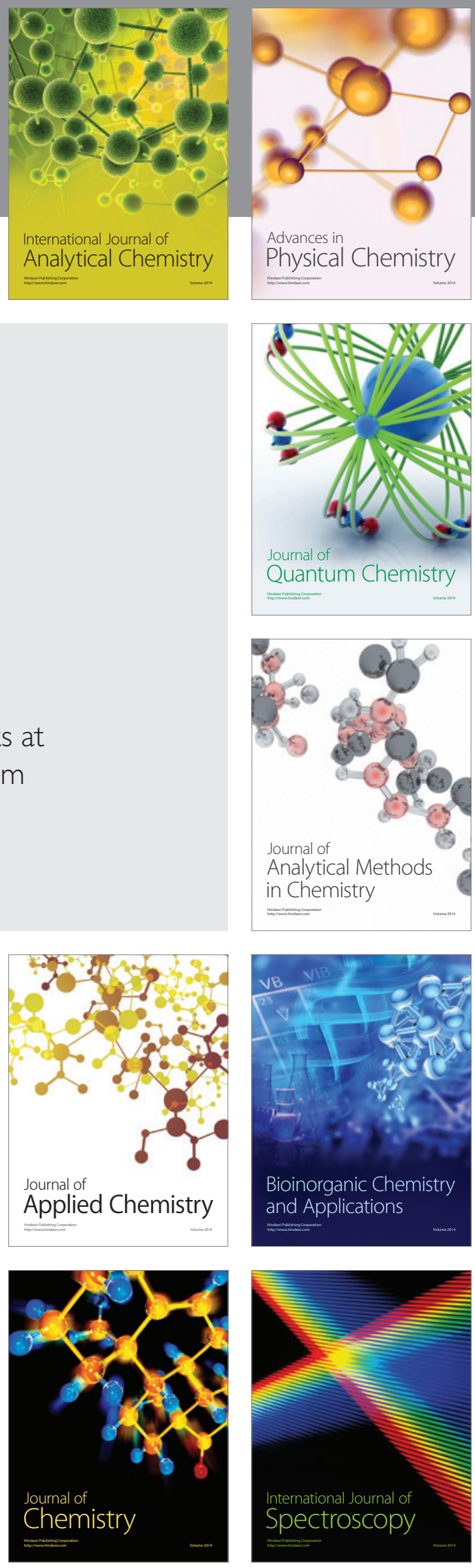